\title{
Cosmologia Quântica com fluido de Van der Waals
}

\author{
F. G. Alvarenga ${ }^{1}$ e S. V. de B. Gonçalves ${ }^{2}$ \\ Universidade Federal do Espírito Santo, ES, Brasil \\ E. V. C. Silva ${ }^{3}$ e G. A. Monerat ${ }^{4}$ \\ Universidade do Estado do Rio de Janeiro, RJ, Brasil \\ M. G. de C. Batista ${ }^{5}$ \\ Universidade Federal de Roraima, RR, Brasil \\ G. de O. Neto ${ }^{6}$ \\ Universidade Federal de Juiz de Fora, MG, Brasil

${ }^{1}$ flavio.alvarenga@ufes.br ${ }^{2}$ sergio.vitorino@pq.cnpq.br
${ }_{4}^{4}$ germano.monerat@ evasquez@uerj.br
${ }^{3}$ enpq.br

\begin{abstract}
Resumo
Propõe-se a quantização de um modelo cosmológico descrevendo o Universo preenchido com um fluido de radiação e um fluido de Van der Waals como componente para explicar a expansão acelerada do Universo. Através do método espectral de Galerkin, os autoestados da equação de Wheeler-DeWitt são obtidos de modo a possibilitar a aplicação da interpretação de vários mundos da Mecânica Quântica. Para certos valores de parâmetros do modelo os resultados preliminares revelam que em nível quântico o Universo não possui singularidade, o estado de tamanho zero e densidade de energia infinita.
\end{abstract}

\section{Introdução}

O Universo é composto prioritariamente pelo setor escuro, $70 \%$ de energia escura, $25 \%$ de matéria escura, restando apenas $5 \%$ na forma de matéria ordinária bariônica [1]. Vários trabalhos têm apresentado o fluido de Van der Waals como candidato à energia escura, seja como componente única ou em cenários em que o mesmo aparece em combinação com outros fluidos [2, 3]. A presença do fluido de Van der Waals interagindo com o campo gravitacional pode simu- lar por exemplo, um período inflacionário onde a aceleração cósmica cresce exponecialmente e o fluido de Van der Waals comporta-se como um campo tipo inflaton; um período acelerado $(a>0)$ mas que decresce tendendo a zero a medida que a densidade de energia do fluido de Van der Waals decai; um período desacelerado que corresponde à era dominada pela matéria com pressão positiva; e o momento atual da expansão acelerada, onde a densidade de energia escura supera a densidade de energia do fluido de Van der Waals. Em [4] tais propostas são mencionadas e um 
extenso guia literário apresentado.

Por outro lado, mesmo o modelo cosmológico mais confiável em termos observacionais, o chamado modelo $\Lambda C D M$ [5, 6] não elimina a sigularidade inicial tipo big bang do Universo no tempo $t=0$. Neste estado as divergências nas grandezas físicas, por exemplo, temperatura e densidade de energias infinitas impedem qualquer descrição do Universo na denominada época de Planck. Como alternativa, a Cosmologia Quântica [7] surge como uma teoria de condições iniciais que possibilita eliminar a singularidade inicial em função dos efeitos quânticos existentes numa época em que o Universo tinha um tamanho muito menor que o núcleo atômico.

Neste cenário formula-se um modelo cosmológico composto de dois fluidos: o fluido de Van der Waals e um fluido de radiação. Os fluidos têm papéis bem distintos, enquanto o primeiro assumirá o papel de dirigir a expansão acelerada do Universo, o segundo predominante nos primórdios no Universo exercerá o papel de tempo na dinâmica decorrente da equação de Wheeler-DeWitt, ao ser introduzido fenomenologicamente através dos potenciais-velocidades de Schutz [8].

O trabalho está dividido da seguinte maneira: na seção 2 o modelo é estabelecido através da construção da Hamiltoniana do sistema Universo com suas contribuições gravitacional e dos fluidos de Van der Waals e radiação; na seção 3 a equação de Wheeler-DeWitt é resolvida pelo método espectral de Galerkin [9].

\section{O Modelo}

A ação de Einstein-Hilbert para o campo gravitacional com o termo de contorno é expressa na forma

$$
S_{g}=\int_{M} d^{4} x \sqrt{-g} R+2 \int_{\partial M} d^{3} x \sqrt{h} K
$$

onde $R$ é o escalar de curvatura, tomado como função da métrica $g_{\mu \nu}$ e suas derivadas, $K=h_{a b} K^{a b}$ tal que $K_{a b}$ é a curvatura extrínseca e $h_{a b}$ é a métrica induzida sobre a hipersuperfíe espacial tridimensional. Acima $\partial M$ é o contorno da variedade quadridimensional $M$.

Para o Universo homogêneo e isotrópico descrito pela métrica de Friedmann-Lemaître-Robertson-Walker $(\operatorname{com} c=1$ e $k=1)$ :

$d s^{2}=-N(t)^{2} d t^{2}+a(t)\left(\frac{d r^{2}}{1-r^{2}}+r^{2} d \Omega\right)$,

em que $N(t)$ é a função lapso, que corresponde à componente normal da quadrivelocidade, e $a(t)$ é o fator de escala do Universo.

Seguindo o formalismo canônico, a inserção da métrica na ação (1) permite identificar a Hamiltoniana gravitacional:

$$
\mathscr{H}_{g}=-\frac{p_{a}^{2}}{24 a}-6 a
$$

A inclusão de conteúdo material na forma de um fluido perfeito de radiação relativístico pode ser efetuada através da representação de sua quadrivelocidade em termos dos potenciais-velocidades $\varepsilon, \theta$ e $S:$

$$
U_{0}=\frac{1}{\mu}(\dot{\varepsilon}+\theta \dot{S}),
$$

cada qual satisfazendo sua própria equação de movimento. $\mathrm{Na}$ expressão acima $\mu$ é a entalpia específica, $S$ é a entropia específica. As variáveis $\varepsilon$ e $\theta$ não têm significado físico claro.

A ação da matéria é especialmente simples:

$$
S_{f}=\int d^{4} x \sqrt{-g} p,
$$

onde $p$ é a pressão, a qual está associada com a densidade de energia através da equação de estado $p=\frac{1}{3} \rho$. As equações de Einstein são obtidas pela variação 
da ação (5) mais a ação de EinsteinHilbert em relação ao tensor métrico, enquanto variações relativas aos potenciaisvelocidades fornecem as equações Eulerianas da evolução do fluido.

Novamente, o formalismo canônico fornece a Hamiltoniana do fluido:

$$
\mathscr{H}_{f}=\frac{p_{\phi}^{\frac{4}{3}}}{a} e^{S} .
$$

As transformações canônicas

$$
\begin{gathered}
T=p_{S} e^{-S} p_{\phi}{ }^{-\frac{4}{3}} ; p_{T}=p_{\phi}{ }^{\frac{4}{3}} e^{S} ; \\
\bar{\phi}=\phi-\frac{4}{3} \frac{p_{S}}{p_{\phi}} ; \bar{p}_{\phi}=p_{\phi} .
\end{gathered}
$$

permitem colocar a Hamiltoniana (6) em uma forma mais sugestiva:

$$
\mathscr{H}_{f}=\frac{p_{T}}{a} .
$$

Em [10, 11] descrições detalhadas dos passos que conduziram à (3) e (8) são apresentadas.

O fluido de Van der Waals pode agora ser introduzido no modelo a partir de sua equação de estado

$$
p_{E E}=\frac{\omega \rho_{E E}}{1-\beta \rho_{E E}}-\alpha \rho_{E E}^{2},
$$

sendo $\omega, \alpha$ e $\beta$ parâmetros livres, enquanto $\rho_{E E}$ é a densidade de energia do fluido escuro.

A conservação do tensor momentoenergia,

$$
\dot{\rho}_{E E}+3 \frac{\dot{a}}{a}\left(\rho_{E E}+p_{E E}\right)=0,
$$

possibilita escrever a densidade de energia em termos do fator de escala do Universo $a$ :

$$
\rho_{E E}=-\frac{\alpha}{\left(\alpha+C_{1} a^{3}\right)^{2}},
$$

onde particularizou-se (9) para o caso $\omega=$ 0 .
Inserindo (11) na densidade Lagrangiana de (5), a transformação de Legendre $(H=$ $\left.\dot{a} p_{a}-L\right)$ indica de forma imediata a Hamiltoniana do fluido escuro de Van der Waals:

$$
\mathscr{H}_{v w}=\frac{\alpha a^{3}}{\left(\alpha+C_{1} a^{3}\right)^{2}} .
$$

Finalmente, (3), (8) e (12) compõem a Hamiltonina total clássica do sistema gravitacional acoplado à matéria no:

$$
\mathscr{H}=-\frac{p_{a}^{2}}{24}-6 k a^{2}+\frac{\alpha a^{4}}{\left(\alpha+C_{1} a^{3}\right)^{2}}+p_{T}
$$

Acima adotou-se o tempo conforme $N=$ $a$.

\section{A Equação de Wheeler- DeWitt}

O procedimento de quantização consiste na aplicação dos operadores $\hat{p}_{a} \rightarrow-i \frac{\partial}{\partial a}$ e $\hat{p}_{T} \rightarrow-i \frac{\partial}{\partial T}$ em 13 de modo a impor a equação de Wheeler-DeWitt, $\hat{\mathscr{H}} \Psi=0$, sobre a função de onda do Universo:

$$
-\frac{1}{24} \frac{\partial^{2} \Psi}{\partial a^{2}}+V_{e f f}(a)=i \frac{\partial \Psi}{\partial t} .
$$

Acima o tempo foi reescalonado para $t=$ $-T$ e um potencial efetivo definido como

$$
V_{e f f}=6 k a^{2}-\frac{\alpha a^{4}}{\left(\alpha+C_{1} a^{3}\right)^{2}},
$$

e exibido na figura 1 .

O método espectral de Galerkin com o pacote SPECTRAL tem sido uma efetiva ferramenta para resolver a equação de Wheeler-DeWitt associada a modelos de Friedmann-Lemaître-RobertsonWalker com fluido perfeito [12, 13]. Como resultado da aplicação a este modelo, as figura 2 e 3 mostram respectivamente, os três primeiros autoestados de dez calculados e a densidade de probabilidade no instante $t=0$ construída pela 


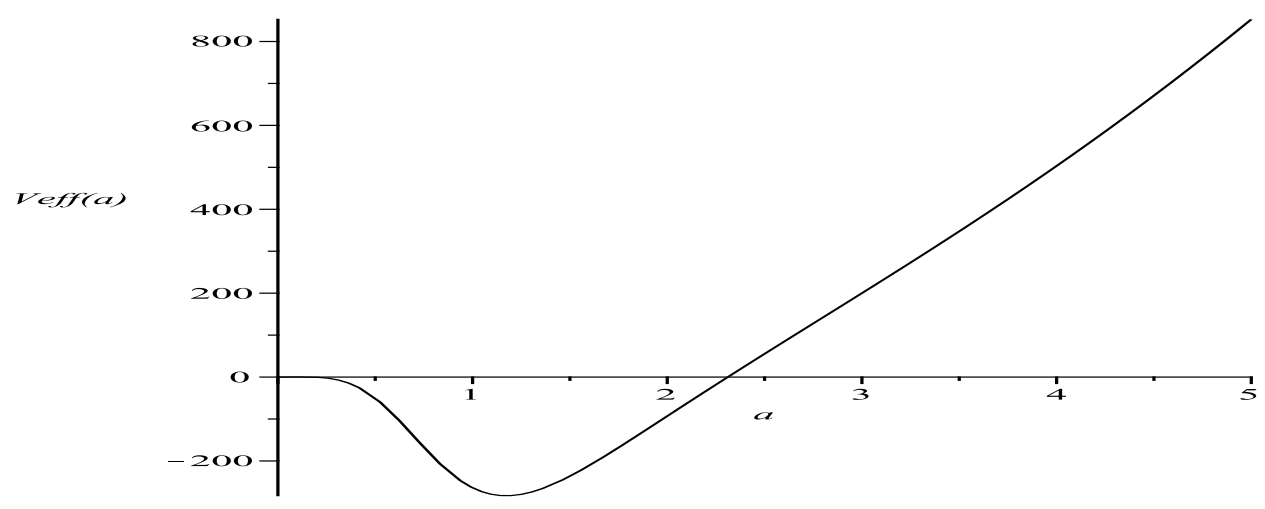

Figura 1: Potencial efetivo para o modelo quântico com fluidos de Van der Waals e radiação.

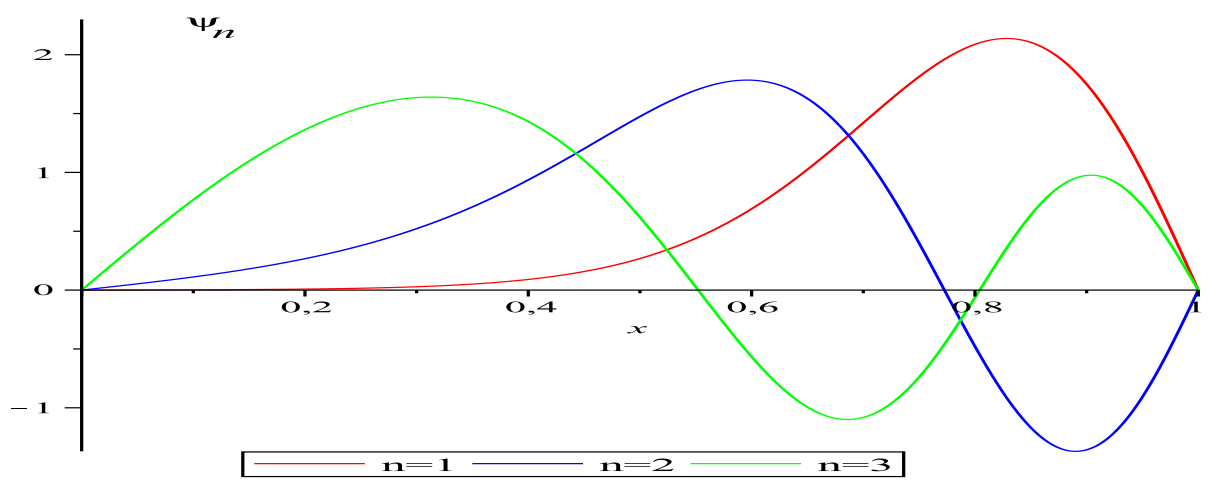

Figura 2: Três primeiros autoestados da função de onda do Universo $\Psi_{n}$.

superposição dos dez primeiros autoestados que geram um pacote de onda que satisfaça a condição de contorno $\Psi(a=$ $0, t)=0$.

$\mathrm{Na}$ interpretação de vários mundos da Mecânica Quântica, o valor esperado do fator de escala pode ser calculado da maneira usual

$$
\langle a\rangle_{t}=\frac{\int_{0}^{\infty} \Psi^{*}(a, t) a \Psi(a, t) d a}{\int_{0}^{\infty} \Psi^{*}(a, t) \Psi(a, t) d a},
$$

o qual (figura 4), revela um Universo que nasce com um tamanho finito $a \neq 0$ antes da fase inflacionária como uma consequência do potencial efetivo (15).

\section{Conclusões}

Efetuou-se no esquema de minisuperespaço a quantização de um modelo cosmológico homogêneo e isotrópico contendo um fluido escuro de Van der Waals e um fluido de radiação. As soluções da equação de Wheeler-DeWitt obtidas pelo método espectral de Galerkin revelam um Universo que nasce com um tamanho finito e portanto não singular no instante $t=0$. O passo seguinte na investigação deste modelo será variar os demais parâmetros $(\omega$ e $\beta$ ) e tentar validá-lo com dados observacionais, por exemplo, condições para entrada na fase inflacionária.

\section{Agradecimentos}

À comissão organizadora do X ECFA pela excelência do evento.

\section{Referências}

[1] V. Mukhanov, Physical Foundations of Cosmology. (Cambridge Univer- 


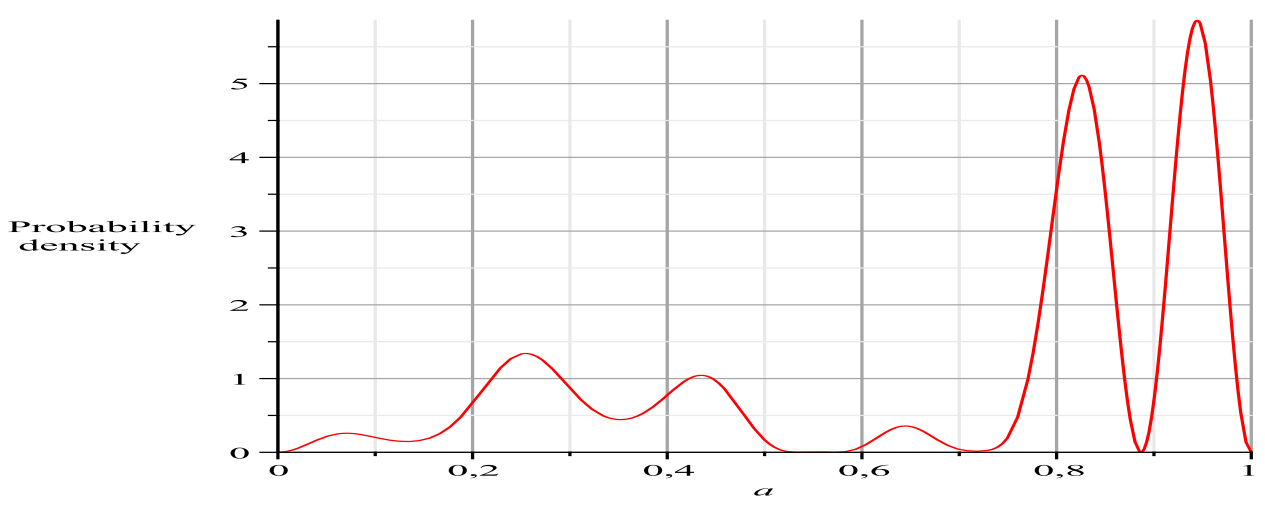

Figura 3: Densidade de probabilidade inicial $|\Psi(a, t=0)|^{2}$.

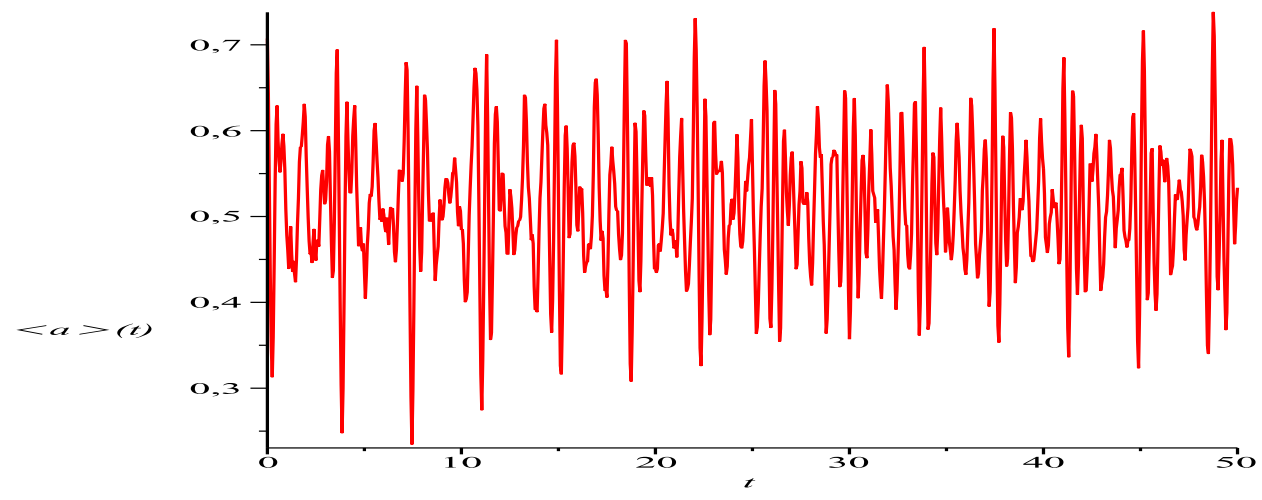

Figura 4: Valor esperado $i_{i}$ do fator de escala do Universo em função do tempo.

sity Press, Singapore, 2005).

[2] S. Capozziello, V. F. Cardone, S. Carloni, S. De Martino, M. Falanga, A. Troisi, e M. Bruni. Journal of Cosmology and Astroparticle Physics JCAP0504:005 (2005).

[3] G. M. Kremer, Phys. Rev. D68, 123507 (2003).

[4] E. Elizalde e M. Khurshudyan, International Journal of Modern Physics D 27, $N^{o} 4,1850037$ (2018).

[5] P. J. E. Peebles, Physical Cosmology. (Princeton University Press, Singapore, 2019).

[6] N. Suzuki e et al. The Astrophysical Journal 746, $N^{o} 1,85$ (2012).

[7] P. Vargas Moniz, Quantum Cosmology - The Supersymmetric Perspective - vol. 1: Fundamentals. (Lect. Notes Phys. 803, 1, 2010).
[8] B. F. Schutz, Phys. Rev. D2, 2762 (1970); D4, 3559 (1971).

[9] J. P. Boyd, Chebyshev and Fourier Spectral Methods, 2nd ed. Dover, New York (2001)

[10] F. G. Alvarenga, R. G. Furtado e S. V. B. Gonçalves, S. Brazilian Journal of Physics, v. 47, p. 96 (2016).

[11] F. G. Alvarenga, J. C. Fabris, N. A. Lemos, G. A. Monerat, Gen. Rel. Grav. 34, 651 (2002).

[12] G. Oliveira-Neto, G.A. Monerat, E.V. Corrêa Silva, C. Neves, L.G. Ferreira Filho, Int. J. Theor. Phys. 52 2991 (2013).

[13] E.V. Corrêa Silva, G.A. Monerat, G. de Oliveira Neto, L.G. Ferreira Filho, Comput. Phys. Commun. 185 380 (2014). 